\title{
Qualidade de vida e fragilidade entre idosos hospitalizados
}

Pedro Martins Faria ${ }^{1}$, Flavia Aparecida Dias ${ }^{2}$, Nayara Paula Fernandes Martins Molina ${ }^{3}$, Janaína Santos Nascimento ${ }^{4}$, Darlene Mara dos Santos Tavares ${ }^{5}$

${ }^{1}$ Enfermeiro. Enfermeiro Hospitalar do Hospital São Francisco. Ribeirão Preto, SP, Brasil. E-mail: pedromartinsfaria@hotmail.com.

${ }^{2}$ Enfermeira, Mestre em Atenção à Saúde. Discente do Programa de Pós-Graduação em Atenção à Saúde - Stricto sensu, nível Doutorado, da Universidade Federal do Triângulo Mineiro. Uberaba, MG, Brasil. Email: flaviadias_ura@yahoo.com.br.

${ }^{3}$ Enfermeira, Mestre em Atenção à Saúde. Discente do Programa de Pós-Graduação em Atenção à Saúde - Stricto sensu, nível Doutorado, da Universidade Federal do Triângulo Mineiro. Uberaba, MG, Brasil. Email: nayara.pfmartins@gmail.com.

${ }^{4}$ Terapeuta Ocupacional, Mestre em Mestre em Atenção à Saúde. Discente do Programa de Pós-Graduação de Ciências Médicas, nível Doutorado, da Universidade do Estado do Rio de Janeiro. Professora Assistente da Universidade Federal do Rio de Janeiro. Rio de Janeiro, RJ, Brasil. E-mail: jananascimento.to@gmail.com.

${ }^{5}$ Enfermeira, Doutora em Enfermagem. Professor Associado da Universidade Federal do Triângulo Mineiro. Uberaba, MG, Brasil. E-mail:

darlenetavares@enfermagem.uftm.edu.br.

Recebido: 23/10/2015.

Aceito: 26/09/2016.
Publicado: 20/12/2016.

\section{RESUMO}

Objetivou-se comparar a qualidade de vida dos idosos hospitalizados segundo o status de fragilidade. Trata-se de um estudo observacional, analítico, transversal, com 255 idosos hospitalizados, no interior de Minas Gerais. Utilizaram-se: instrumento estruturado para os dados socioeconômicos e clínicos, fenótipo de fragilidade de Fried, WHOQOL-BREF e WHOQOL-OLD. Realizou-se análise descritiva e teste Anova-F $(p<0,05)$. Independente do status de fragilidade prevaleceu o sexo masculino, $60 \vdash 70$ anos, casados, que residiam acompanhados e possuíam renda individual de um salário mínimo. Para escolaridade, entre os frágeis predominou de $1 \vdash 4$ anos de estudo; para os préfrágeis e não-frágeis, $4 \vdash 8$ anos. Os frágeis apresentaram: escores significativamente inferiores nos domínios físico e meio ambiente e faceta participação social, comparados aos demais, e; escore significativamente inferior na faceta funcionamento dos sentidos em relação aos pré-frágeis. Ressalta-se a importância de direcionar ações em saúde direcionadas aos itens mais impactados dentre aqueles com fragilidade.

Descritores: Qualidade de Vida; Idoso Fragilizado; Hospitalização.

\section{INTRODUÇÃO}

A população idosa vem crescendo de maneira rápida no país ao Nascimento JS, Tavares DMS. Qualidade de vida e fragilidade entre idosos hospitalizados. Rev. Eletr. Enf. [Internet]. 2016 [acesso em:_____];18:e1195. Disponível em: http://dx.doi.org/10.5216/ree.v18.38214. dos anos ${ }^{(1)}$. Neste panorama, várias condições podem gerar eventos adversos à saúde, dentre eles a fragilidade, definida como "uma síndrome biológica que leva à queda de reserva e da resistência a 
estressores sendo resultado cumulativo do declínio de múltiplos sistemas fisiológicos, causando vulnerabilidade e efeitos adversos"(2).

Há poucos estudos brasileiros que abordam a fragilidade dos idosos no ambiente hospitalar. Em pesquisa realizada no hospital de Passo Fundo-RS a prevalência de idosos frágeis representou 46,5\% e préfrágeis, $49,5 \%{ }^{(3)}$. Investigação conduzida em Ribeirão Preto-SP verificou que 95,0\% dos idosos hospitalizados eram frágeis ${ }^{(4)}$. No âmbito internacional, investigação realizada na Índia, seguindo os critérios proposto por Fried, verificou que $33,2 \%$ dos idosos hospitalizados eram frágeis e $66,8 \%$ não-frágeis ${ }^{(5)}$.

Os possíveis desfechos adversos decorrentes da fragilidade podem deteriorar a QV do idoso ${ }^{(6)}$. Outrossim, estes desfechos podem ser agravados e desencadeados pelo processo de hospitalização. Pesquisa realizada em hospital dos Estados Unidos observou que idosos frágeis possuíam QV inferior nos domínios mental e físico do questionário Short Form-36 (SF-36), quando comparados aos pré-frágeis e não frágeis ${ }^{(6)}$.

A literatura inclui também pesquisas que descrevem a influência adversa da síndrome de fragilidade na QV de idosos em cuidados de saúde primários ${ }^{(7)}$ e naqueles hospitalizados com condições específicas, como síndrome coronariana aguda ${ }^{(8)}$ e insuficiência cardíaca ${ }^{(9)}$. Ressalta-se que o idoso frágil apresenta maior risco de resultados adversos para sua saúde ${ }^{(2)}$, o que reforça a necessidade de outras investigações sobre a temática, permitindo ampliar o conhecimento sobre a relação da fragilidade com a QV em idosos hospitalizados, independente de sua condição clínica, e subsidiar o processo de implementação do diagnóstico e de intervenções específicas com esta população.

Na presente pesquisa será adotado o conceito de QV da Organização Mundial de Saúde que a define como: "a percepção do indivíduo de sua posição na vida no contexto da cultura e sistema de valores nos quais ele vive e em relação aos seus objetivos, expectativas, padrões e preocupações" ${ }^{(10)}$.

Assim, este estudo objetivou comparar a QV dos idosos hospitalizados segundo o status de fragilidade (frágeis, pré-frágeis e não-frágeis).

\section{MÉTODOS}

Pesquisa com abordagem quantitativa, transversal, observacional conduzida nos setores de Clínica Médica e Cirúrgica de um hospital de ensino no interior de Minas Gerais.

Para o cálculo do tamanho amostral, considerou-se a prevalência de fragilidade entre idosos de 30,0\%, considerando outros estudos com idosos em ambiente hospitalar $(33,2 \%)^{(5)}(37 \%)^{(11)}$. Com precisão de $5 \%$ e intervalo de confiança de $95 \%$, para uma população finita de 1.455 idosos elegíveis, chegou-se a uma amostra de 255 idosos. O processo de recrutamento foi por amostragem aleatória sistemática com intervalo de k=2.

Foram incluídos na amostra os idosos com 60 anos ou mais de idade e sem declínio cognitivo. Aqueles idosos que apresentaram restrição para deambular no momento pós-cirúrgico, foram entrevistados no dia seguinte. Foram excluídos idosos que apresentavam sequelas graves de acidente vascular encefálico (AVE) com perda localizada de força e afasia; doença de Parkinson em estágio grave ou instável com associação de comprometimentos graves da motricidade, da fala ou da afetividade que impossibilitasse a realização das 
avaliações; aqueles em estágio terminal e com déficit grave de visão e audição, re-hospitalizados que já haviam sido entrevistados na primeira internação e com restrição para deambular e conversar.

Os dados foram coletados no período de abril de 2013 a março de 2014, momento em que foram abordados 445 idosos; destes, 255 foram entrevistados, dos quais 97 estavam internados na Clínica Médica e 158 na Cirúrgica. Os demais não compuseram a amostra por: recusas (75), declínio cognitivo (101) e outros motivos (14).

Os entrevistadores selecionados tinham experiência prévia em coleta de dados e foram treinados nos aspectos técnico e ético da pesquisa. Contou-se também com um assistente de pesquisa que era responsável por triar os idosos e supervisionar os entrevistadores durante a coleta dos dados.

Antes de iniciar a entrevista aplicou-se o Mini Exame de Estado Mental (MEEM) ${ }^{(12)}$ sendo o ponto de corte para declínio cognitivo: 13 pontos para analfabetos, 18 pontos ou menos para aqueles entre um a 11 anos de estudo e 26 pontos para escolaridade superior a 11 anos $^{(12)}$.

Para coleta dos dados sociodemográficos e as morbidades autorreferidas utilizou-se instrumento estruturado elaborado pelo Grupo de Pesquisa em Saúde Coletiva da Universidade Federal do Triângulo Mineiro. Para a avaliação da QV utilizaram-se dois instrumentos, um genérico denominado World Health Organization Quality of Life - BREF (WHOQOL-BREF) validado no Brasil sendo composto por quatro domínios (físico, psicológico, relações sociais, meio ambiente) ${ }^{(13)}$ e um específico para o idoso, World Health Organization Quality of Life Assessment for Older Adults (WHOQOL-OLD), adaptado no Brasil e constituído por seis facetas (funcionamento dos sentidos; autonomia; atividades passadas, presentes e futuras; participação social; morte e morrer e intimidade) $)^{(14)}$.

A síndrome da fragilidade foi identificada por meio dos cinco componentes do fenótipo de fragilidade propostos por Fried et al $^{(2)}$, conforme segue: perda de peso não intencional; força muscular: verificada com base três medidas na força de preensão palmar; exaustão autorrelatada ${ }^{(15)}$; velocidade de marcha (em segundos) para percorrer 4,6 metros; nível de atividade física ${ }^{(16)}$. Os idosos foram classificados como ativos, quando dispendiam 150 minutos ou mais em atividades física semanalmente, e em inativos, quando realizavam de zero a 149 minutos de atividades físicas por semana conforme as diretrizes da American Heart Association e American College of Sports Medicine ${ }^{(17)}$. Foram classificados como frágeis os idosos com três ou mais itens; pré-frágeis quando apresentavam um ou dois itens, e; e não frágeis ou robustos quando não apresentavam nenhum item ${ }^{(2)}$.

As variáveis estudadas foram: sexo, faixa etária, estado conjugal, arranjo domiciliar, escolaridade, renda individual mensal, fragilidade e QV.

Os dados foram processados no microcomputador em uma planilha de Excel ${ }^{\bullet}$ em dupla entrada. Ao término, verificou a consistência entre as duas bases de dados, procedendo a correção, baseada na entrevista original, quando necessário.

Para a análise descritiva foi utilizada distribuição de frequência e para a comparação das variáveis de interesse o teste Anova-F, sendo o nível de significância $(\alpha)$ de $5 \%$ e os testes considerados significativos 
quando $p<\alpha$. Cada domínio do WHOQOL-BREF e faceta do WHOQOL-OLD foram analisados isoladamente com as respectivas sintaxes. 0 escore varia de zero a 100, sendo que o maior número corresponde à melhor QV.

Este projeto foi aprovado pelo Comitê de Ética em Pesquisa da Universidade Federal do Triângulo Mineiro, protocolo $n^{\circ}$ 2.511. A entrevista foi conduzida após a assinatura do Termo de Consentimento Livre e Esclarecido pelo idoso.

\section{RESULTADOS}

No presente estudo, $26,3 \%$ dos idosos foram classificados como frágeis, $53,3 \%$ pré-frágeis e $20,4 \%$ não-frágeis.

Nos três grupos, frágeis, pré-frágeis e não frágeis, houve prevalência de homens (50,7\%, 64\%, 67,3\%), $60 \vdash 70$ anos $(52,2 \%, 61,8 \%, 73,1 \%)$, casados ou que moravam com companheiro $(47,8 \%, 67,6 \%, 61,5 \%)$, que residiam acompanhados $(83,6 \%, 86,8 \%, 80,8 \%)$ e possuíam renda individual de um salário mínimo (64,2\%, $55,9 \%, 48,1 \%)$; para a escolaridade, entre os frágeis predominou de $1 \vdash 4$ anos de estudo $(32,8 \%)$, já para os pré-frágeis (37,5\%) e não-frágeis (38,5\%) $4 \vdash 8$ anos.

Os idosos frágeis relataram ter QV nem ruim nem boa (43,3\%); já os pré-frágeis (48,5\%) e não-frágeis $(59,6 \%)$ referiram como boa. Nos três grupos os idosos estavam satisfeitos com a própria saúde representando $38,8 \%$ dos frágeis, $44,4 \%$ entre os pré-frágeis e $53,8 \%$ para os não-frágeis.

Os idosos frágeis apresentaram escores médios significativamente inferiores nos domínios físico $(p<0,001)$ e meio-ambiente $(p=0,001)$ em relação aos pré-frágeis e não-frágeis (Tabela 1$)$.

No psicológico apesar do teste Anova-F ter sido estatisticamente significativo $(p=0,044)$, no qual os frágeis obtiveram escores inferiores, as comparações múltiplas não indicaram diferenças entre os escores médios dos grupos definidos no estudo (Tabela 1).

Os idosos frágeis apresentaram menor escore médio no funcionamento dos sentidos quando comparados aos pré-frágeis $(p=0,030)$. Na participação social, os frágeis obtiveram escore médio significativamente inferiores aos pré-frágeis e não-frágeis $(p<0,001)$ (Tabela 1$)$.

Tabela 1. Distribuição dos escores dos domínios do WHOQOL-BREF e facetas do WHOQOL-OLD, segundo o status de fragilidade de

\begin{tabular}{|c|c|c|c|c|c|c|c|c|}
\hline \multirow{2}{*}{ Variáveis } & \multicolumn{2}{|c|}{ Frágeis } & \multicolumn{2}{|c|}{ Pré-frágeis } & \multicolumn{2}{|c|}{ Não-frágeis } & \multirow{2}{*}{$\mathbf{F}$} & \multirow{2}{*}{$p$} \\
\hline & Média & DP & Média & DP & Média & DP & & \\
\hline \multicolumn{9}{|l|}{ WHOQOL-BREF } \\
\hline Físico & 47,55 & 16,54 & 59,69 & 14,97 & 62,16 & 12,64 & 18,639 & $<0,001$ \\
\hline Psicológico & 63,12 & 18,05 & 67,37 & 13,59 & 69,55 & 11,68 & 3,167 & 0,044 \\
\hline Relações sociais & 70,02 & 18,12 & 74,45 & 15,29 & 71,47 & 13,03 & 1,981 & 0,140 \\
\hline Meio ambiente & 55,69 & 13,61 & 62,27 & 11,63 & 62,14 & 11,69 & 7,119 & 0,001 \\
\hline \multicolumn{9}{|l|}{ WHOQOL-OLD } \\
\hline Funcionamento dos sentidos & 60,35 & 23,78 & 68,61 & 22,46 & 69,95 & 22,89 & 3,572 & 0,030 \\
\hline Autonomia & 61,38 & 15,73 & 64,75 & 16,56 & 66,59 & 13,44 & 1,752 & 0,175 \\
\hline Atividades passadas, presentes e futuras & 69,59 & 12,35 & 71,05 & 16,80 & 68,63 & 15,23 & 0,526 & 0,591 \\
\hline Participação social & 57,00 & 16,87 & 66,08 & 15,55 & 67,31 & 12,04 & 9,525 & $<0,001$ \\
\hline Morte e morrer & 70,24 & 26,38 & 71,60 & 26,48 & 70,79 & 26,13 & 0,063 & 0,939 \\
\hline Intimidade & 71,92 & 20,60 & 73,53 & 19,65 & 67,19 & 19,56 & 1,914 & 0,150 \\
\hline
\end{tabular}

Rev. Eletr. Enf. [Internet]. 2016 [acesso em:___/_];18:e1195. Disponível em: http://dx.doi.org/10.5216/ree.v18.38214. 


\section{DISCUSSÃO}

A prevalência do status de fragilidade desta investigação foi inferior ao constatado em um hospital brasileiro de Passo Fundo-RS em relação aos frágeis $(46,5 \%)$ e semelhante aos pré-frágeis $(49,5 \%)^{(3)}$. Uma das causas para a diferença em relação aos frágeis pode ser pelo fato do estudo em Passo Fundo-RS contemplar idosos de 65 anos ou mais, bem como possíveis diferenças regionais.

Em comparação com estudos internacionais, os percentuais foram semelhantes em estudos com frágeis e pré-frágeis em um hospital da Bélgica $\left(32,3 \%\right.$ e $52 \%$, respectivamente) ${ }^{(18)}$, e dos Estados Unidos $(21,5 \% \text { e } 54,7 \% \text {, respectivamente })^{(6)}$. Tal fato pode estar expressando a semelhança no perfil dos idosos e/ou métodos de inclusão do presente estudo, com as investigações internacionais. Deste modo, é necessário que esta condição seja monitorada nos serviços de saúde nos diversos níveis de atenção e sejam desenvolvidas atividades preventivas.

Diante destes dados, torna-se necessário a articulação entre a equipe multiprofissional de saúde em todos os níveis de atenção, com intuito de detectar precocemente os sinais da condição de fragilidade e traçar intervenções que minimizem a sua ocorrência.

Independente dos status de fragilidade houve prevalência de homens na amostra, assim como em estudo com idosos hospitalizados de Ribeirão Preto-SP $(60,7 \%)^{(4)}$. No entanto, em investigação realizada com idosos hospitalizados em Passo Fundo-RS prevaleceram as mulheres $(50,5 \%)^{(3)}$. O predomínio do sexo masculino, pode estar relacionado a que homens tendem a menor cuidado com a própria saúde ${ }^{(19)}$ tornandoo mais suscetível à hospitalização pelo possível agravamento da sua condição de saúde.

Quanto à idade, independente do status de fragilidade, prevaleceu os idosos mais jovens. Já em estudo nacional, com idosos hospitalizados, predominou a faixa etária de 70 a 79 anos para fragilidade leve e 80 anos ou mais para fragilidade severa ${ }^{(4)}$. No entanto, evidenciou-se maior percentual de idosos frágeis nas maiores faixas etárias.

Sabe-se que com o avançar dos anos, os idosos apresentam alterações fisiológicas próprias do processo de envelhecimento que os tornam mais susceptíveis ao aparecimento de comorbidades e ao declínio da capacidade física ${ }^{(5-6)}$, que têm sido associados à síndrome de fragilidade ${ }^{(2)}$, podendo justificar os dados do presente inquérito. Desta forma, durante o processo de hospitalização o enfermeiro pode atuar na identificação e na prevenção das manifestações clínicas e dos possíveis agravos entre os idosos principalmente dentre aqueles mais jovens.

A prevalência de casados independente do status de fragilidade corrobora com estudo hospitalar de Ribeirão Preto-SP ${ }^{(4)}$. Fato importante do presente estudo é o percentual relevante de viúvos entre os frágeis. A morte do cônjuge costuma gerar estresse e sofrimento ao idoso ${ }^{(20)}$, possibilitando o desencadear de consequências negativas para o seu aspecto físico, levando a maior propensão à morbidades; o que poderia favorecer o processo de fragilização ${ }^{(3,21)}$. Com isto torna-se importante o suporte social pelos familiares, amigos ou grupos, e equipe de saúde multidisciplinar. Nesse sentido, é relevante que o profissional de saúde, identifique a rede de suporte do idoso, em especial aqueles sem companheiro. 
Em relação ao arranjo domiciliar, mesmo a maioria vivendo acompanhado é importante destacar o percentual de idosos que vivem sós e se encontram em situação de fragilidade ou pré-fragilidade; conjuntura esta, que pode impor aos idosos maiores dificuldades na realização das suas atividades cotidianas e nos seus cuidados com a sua saúde. Ressalta-se então a necessidade do rastreamento pelas unidades de saúde, visando realizar atividades direcionadas ao acompanhamento bem como buscar estratégias de apoio social.

Quanto a escolaridade, os dados desta pesquisa condizem com inquérito realizado com idosos hospitalizados de Passo Fundo-RS, onde independente do nível de fragilidade prevaleceram os alfabetizados ${ }^{(3)}$. Vale ressaltar que a presente pesquisa demonstra entre os idosos analfabetos, maior porcentagem de frágeis. Ainda que não justifique o resultado, espera-se que idosos com maiores níveis de escolaridade possuam maior acesso à informação e aos serviços de saúde; itens que podem contribuir com a manutenção da saúde do idoso possivelmente minimizando a ocorrência de fragilidade.

A maior renda entre aqueles sem fragilidade, não condiz com estudo realizado em hospital de Passo Fundo-RS, que obteve maior renda entre os frágeis ${ }^{(3)}$. A maior renda pode propiciar uma melhor percepção por cuidados preventivos, autocuidado ${ }^{(3)}$. Enquanto que a menor renda pode comprometer o acesso do idoso aos serviços de saúde podendo contribuir para maximização dos agravos e consequente processo de fragilização.

O predomínio do relato de QV nem ruim nem boa entre os frágeis, e boa entre os pré-frágeis e não frágeis, pode estar relacionado ao fato dos idosos frágeis apresentarem piores níveis de saúde, renda, alimentação, menos sentimentos positivos, autoavaliação de saúde ruim ${ }^{(22)}$.

A menor satisfação dos frágeis com a própria saúde é condizente com inquérito em Ribeirão Preto-SP no qual os idosos hospitalizados relataram sua saúde como ruim ${ }^{(4)}$. O menor índice de satisfação com a própria saúde dos frágeis em relação aos pré-frágeis e não frágeis, pode estar relacionado ao maior declínio das funções corporais ${ }^{(2)}$, refletindo negativamente na autoavaliação da saúde.

Os menores escores de QV no domínio físico entre os frágeis são semelhantes ao encontrado em estudo nos Estados Unidos, porém utilizando o SF-36 ${ }^{(6)}$. Tal achado pode ser explicado pelo fato do idoso frágil ser mais propenso a efeitos adversos na sua saúde ${ }^{(2)}$, contribuindo para dor e desconforto ${ }^{(13)}$, mensurados neste domínio. Soma-se o possível maior desenvolvimento de comorbidades $^{(3)}$ favorecendo a dependência de medicamentos e tratamentos. Salienta-se ainda, que a hospitalização contribui para a dor e a fadiga bem como diminuição da qualidade do sono do idoso devido à luz excessiva, durante o recebimento de cuidados de enfermagem ${ }^{(19)}$, podendo impactar negativamente na QV especialmente daquele com piores condições de saúde.

Nesse contexto, é relevante refletir acerca do papel da equipe de enfermagem no controle e mensuração da dor, além de fornecer subsídios para a equipe médica determinar o adequado tratamento para o seu alívio. Sugere-se ainda que a programação da assistência, em especial no período noturno, possa contribuir com o indivíduo hospitalizado a manter o sono de qualidade.

No domínio psicológico os frágeis apresentaram menores escores de QV, corroborando com estudo 
conduzido nos Estados Unidos, o qual verificou entre os idosos piores escores relacionados ao componente mental $^{(6)}$. Tal fato pode estar relacionado aos sintomas depressivos, que tendem a ser mais frequentes entre os frágeis afetando negativamente seu aspecto psicológico ${ }^{(18)}$. Outro fator que pode estar impactando neste domínio de QV são os piores escores no aspecto físico, que poderiam contribuir para o advento de sentimentos negativos relacionados à sua condição de saúde.

Considerando ainda que este domínio avalia a espiritualidade, religiosidade e crenças pessoais somase a possibilidade dos profissionais identificarem, de forma ética e com respeito estes aspectos, tendo em vista que são recursos que contribuem para o enfrentamento do adoecimento e para as mudanças ocasionadas pela hospitalização ${ }^{(23)}$.

Diante disso, os profissionais de saúde devem oferecer cuidados a estes idosos além das suas manifestações clínicas e relacionadas a hospitalização. Deve-se considerar as implicações emocionais e os sentimentos negativos relacionados a este processo. Além disso, quando possível, estimular o apoio e a participação da família e de amigos como forma de suporte, pois contribuem para a adaptação do idoso na rotina hospitalar e no enfrentamento da hospitalização(23).

Quanto ao domínio meio ambiente, infere-se que o processo de hospitalização possa influenciar na segurança física, proteção e participação e oportunidade de recreação/lazer ${ }^{(13)}$, que são itens avaliados neste domínio; assim devido a piores condições físicas o idoso frágil teria maior impacto nestes aspectos. Para que o idoso venha a se sentir seguro e protegido é importante que a equipe de enfermagem ofereça suporte de modo individualizado considerando as suas especificidades ${ }^{(23)}$.

Recomenda-se desenvolver atividades de lazer que possam ser executadas no hospital e sejam de interesse dos idosos, podendo priorizar aquelas possíveis de serem realizadas em domicílio após a alta hospitalar.

A menor média na faceta funcionamento dos sentidos entre os frágeis corrobora com dados de pesquisa com idosos das comunidades do México $^{(24)}$, que encontrou piores níveis de audição e acuidade visual relacionados a fragilidade. A maior prevalência de deficiência auditiva tem sido encontrada entre idosos mais velhos e com deficiência visual ${ }^{(25)}$. Este fato pode ter contribuído para o presente resultado considerando que nesta pesquisa a maior proporção de idosos mais velhos esteve entre os frágeis.

O menor escore de QV na faceta participação social para os frágeis está em consonância com pesquisa hospitalar dos Estados Unidos ${ }^{(6)}$. A menor capacidade física e pior funcionamento sensorial dos frágeis pode estar limitando a participação em atividades. Nesse contexto, a equipe de saúde deve identificar as preferências dos idosos, de acordo com as incapacidades físicas e sensoriais e utilizar as opções existentes dentro do hospital; como salas ecumênicas, de convivência e lazer. Assim, pode-se ampliar a interação com os profissionais de saúde, familiares e demais indivíduos hospitalizados favorecendo a ampliação de atividades sociais, impactando positivamente na QV. 


\section{CONCLUSÃO}

Os idosos frágeis apresentaram escores significativamente inferiores de QV considerando-se os diversos domínios e facetas. Nesta perspectiva, podem ser repensadas estratégias em saúde direcionadas aos itens mais impactados dentre aqueles com fragilidade.

O estudo apresenta como limitação o delineamento transversal impossibilitando apresentar a causalidade entre QV e síndrome de fragilidade, sendo sugeridos novos estudos com outros recortes. Salienta-se que a utilização de instrumento específico para esta faixa etária compreende aspectos condizentes com o conceito de QV delineado para esta população e que não foram contemplados em investigações nacionais e internacionais anteriores, dificultando a discussão dos resultados.

Acredita-se que o presente inquérito contribua para reflexão acerca dos fatores que afetam a QV de acordo com o status de fragilidade, viabilizando subsídios para o delineamento e implantação de estratégias de cuidados a fim de minimizar este impacto.

\section{REFERÊNCIAS}

1. Instituto Brasileiro de Geografia e Estatística (IBGE). Vou te contar. A revista do CENSO. Avaliação 2010: a coleta, os primeiros resultados e as próximas divulgações. [Internet] 2011 [acesso em: 08 mai 2015]. Disponível em:

http://censo2010.ibge.gov.brimages/pdf/censo2010/revista/vtc20_web.pdf.

2. Fried LP, Tangen CM, Walston J, Newman AB, Hirsch C, Gottdiener J, et al. Frailty in older adults: evidence for a phenotype. J Gerontol A Biol Sci Med Sci. 2001; 56(3):146-56.

3. Oliveira DR, Bettinelli LA, Pasqualotti A, Corso D, Brock F, Erdmann AL. Prevalência de síndrome da fragilidade em idosos de uma instituição hospitalar. Rev Lat Am Enfermagem. [Internet] 2013 [acesso em: 10 jul 2015];21(4): [8 telas]. Disponível em: http://www.scielo.br/pdf/rlae/v21n4/pt 0104-1169-rlae-21-04-0891.pdf.

4. Storti LB, Fabrício-Whebe SCC, Kusumota L, Rodrigues RAP, Marques S. Fragilidade de idosos internados na clínica médica da unidade de emergência de um hospital geral terciário. Texto e Contexto Enfermagem. 2013;22(2):452-9.

5. Khandelwal D, Goel A, Kumar U, Gulati V, Narang R, Dey AB. Frailty is associated with longer hospital stay and increased mortality in hospitalized older patients. J Nutr. 2012;16(8):732-35.

6. Gharacholou SM, Roger VL, Lennon RJ, Rihal CS, Sloan JA, Spertus JA, et al. Comparison of frail patients versus nonfrail patients $\geq 65$ years of age undergoing percutaneous coronary intervention. Am J Cardiol. 2012;109(11):156975.

7. Lenardtl MH, Carneiro NHK, Binottol MA, Willig MH, Lourenço TM, Albino J. Frailty and quality of life in elderly primary health care users. Rev bras enferm. 2016;69(3):478-83.

8. Lisiak M, Uchmanowicz, I, Wontor R. Frailty and quality of life in elderly patients with acute coronary syndrome. Clin Interv Aging. 2016;11(1):553-62.

9. Uchmanowicz I, Gobbens R JJ. The relationship between frailty, anxiety and depression, and health-related quality of life in elderly patients with heart failure. Clin Interv Aging. 2015; 10(1):1595-1600.

10. The WHOQOL GROUP. The World Health Organization quality of life assessment: position paper from the World Health Organization. Soc Sci Med. 1995;41(10):1403-9.

11. Purser JL, Kuchibhatla MN, Fillenbaum GG. Identifying frailty in hospitalized older adults with significant coronary artery disease. JAGS. 2006 Nov; 54(11):1674-81.

12. Bertolucci PHF, Brucki SMD, Campacci SR, Juliano Y. O mini-exame do estado mental em uma população geral: impacto da escolaridade. Arq Neuropsiquiatria. 1994;52(1):1-7.

13. Fleck MPA, Louzada S, Xavier M, Chachamovich E, Vieira G, Santos L. Aplicação da versão em português do instrumento abreviado de avaliação da qualidade de vida "WHOQOL-bref". Rev Saude Publica. 2000;34(2):178-83. 14. Fleck MPA, Chachamovich E, Trentini CM. Development and validation of the Portuguese version of the WHOQOL- 
OLD module. Rev Saude Publica. 2006;40(5):785-91.

15. Batistoni ST, Neri AL, Cupertino APFB. Validade da escala de depressão do Center for Epidemiological Studies entre idosos brasileiros. Rev Saude Publica. 2007;41(4):598-605.

16. Benedetti TRB, Mazo GZ, Barros MVG. Aplicação do questionário internacional de atividades físicas (IPAQ) para avaliação do nível de atividades físicas de mulheres idosas: validade concorrente e reprodutibilidade teste-reteste. Revista Brasileira de Ciência e Movimento. 2004;12(1):25-33.

17. Pate RR, Pratt M, Blair SN, Haskell WL, Macera CA, Bouchard C, et al. Physical activity and public health. A recommendation from the Centers for Disease Control and Prevention and the American College of Sports Medicine. JAMA. 1995;273(5):402-7.

18. Joosten E, Demuynck M, Detroyer E, Miisen K. Prevalence of frailty and its ability to predict in hospital delirium, falls, and 6-month mortality in hospitalized older patients. BioMed Central. 2014;14:1.

19. Costa SV, Ceolim MF. Fatores que interferem na qualidade do sono de pacientes internados. Rev Esc Enferm USP. 2013;47(1):46-52.

20. Galicioli TGP, Lopes ESL, Rabelo DF. Superando a viuvez na velhice: o uso de estratégias de enfrentamento. Revista Temática Kairós Gerontologia. 2012;15(4):225-37.

21. Vieira RA, Guerra RO, Giacomin KC, Vasconcelos KSS, Andrade ACS, Pereira LSM, et al. Prevalência de fragilidade e fatores associados em idosos comunitários de Belo Horizonte, Minas Gerais, Brasil: dados do Estudo FIBRA. Cad Saude Publica. 2013;29(8):1631-43.

22. Mello AC, Engstrom EL, Alves LC. Fatores sociodemográficos e de saúde associados à fragilidade em idosos: uma revisão sistemática de literatura. Cad Saude Publica. 2014;30(6):1-25.

23. Caldas CP, Teixeira PC. O idoso hospitalizado sob o olhar da teoria de enfermagem humanística. Ciência, Cuidado e Saúde. 2012;11(4):748-57.

24. González EDL, Pérez HET, Hermosillo HG, Rodríguez JAC, Torres G. Fragilidad y su asociación con mortalidad, hospitalizaciones y dependencia funcional en mexicanos de 60 años o más. Medicina Clinica. 2012;138(11):468-74. 25. Cruz MS, Lima MCP, Santos JLF, Duarte YAO, Lebrão ML, Ramos-Cerqueira ATA. Deficiência auditiva referida por idosos no Município de São Paulo, Brasil: prevalência e fatores associados (Estudo SABE, 2006). Cad Saude Publica. 2012;28(8):1479-92. 